Proceedings of the International School and Conference on Optics and Optical Materials, ISCOM07, Belgrade, Serbia, September 3-7, 2007

\title{
Surface Plasmon Frequency Spectrum in a System of Two Spherical Dielectric Coated Metallic Nanoparticles
}

\author{
T. MAKARYAN ${ }^{a}$, A. Melikyan ${ }^{b, *}$ AND H. Minassian $^{c}$ \\ ${ }^{a}$ Faculty of Radiophysics, Yerevan State University, Armenia \\ ${ }^{b}$ Russian-Armenian (Slavonic) State University, Yerevan, Armenia \\ ${ }^{c}$ Yerevan Physics Institute after A. Alikhanyan, Yerevan, Armenia \\ Semi-analytical method of surface plasmon frequency calculation for the \\ system of two almost touching coupled dielectric coated metallic nanospheres \\ is presented. The method allows transforming the problem to numerical so- \\ lution of two simple algebraic equations for arbitrary values of parameters \\ - particle radius, distance between sphere centers, dielectric permittivity \\ of the matrix, dielectrics and metals. It is especially easy to get the ob- \\ vious graphical solution. The surface plasmon frequencies of longitudinal \\ and transversal oscillations of the system are calculated and good agreement \\ with the experimental results is achieved.
}

PACS numbers: 73.22.-f

\section{Introduction}

The calculation of surface plasmon (SP) frequencies of metallic nanoparticles (MNP) with size less than SP wavelength can be reduced to the electrostatic boundary problem. It is well known that this problem is solved analytically only for two cases: sphere and spheroids. For more complicated shapes special approximate methods are developed to determine the SP resonance frequencies as a function of particle shape and size [1-4]. The problem becomes more complicated for a pair or array of closely spaced MNPs that are currently of great interest due to their applications in surface enhanced Raman scattering, nanotechnology, and biosensors [5-8]. The main peculiarity of these systems is conditioned by the effect of near-field interaction leading to the significant changes of SP spectra [5, 6, 9-16].

For the calculation of plasmon resonances of coupled particles the boundary integral method is employed $[17,18]$. This approach has the advantage that in

*corresponding author; e-mail: amelikyan@shen.am 
numerical calculations with the use of the finite elements method only the particle surface is discretized instead of discretizing its complete volume. The aim of discretization is reducing the integral equation to the algebraic eigenvalue problem for SP modes. This procedure, however, still requires substantial computing time as to reach satisfactory accuracy it is necessary to divide the boundaries into very large number of elements. Also there are many parameters determining the SP frequency, that increase the computing time: the interparticle distance, dielectric function of the bulk metal that in case of noble metals accounts for the $d$-electron contribution, plasma frequency for bulk metal, and the dielectric constant of host matrix. To overcome above-mentioned difficulty, we have developed an analytical approach in the frame of boundary integral method and presented in this paper. The approach allows easy obtaining of the SP frequencies of two coupled identical spheres for arbitrary values of all parameters. In our approach, we neglect the retardation effects meaning that the particle size and the interparticle distance are smaller than the plasmon wavelength, which takes place in various experiments $[10,19]$.

\section{Calculation of SP frequencies}

In this section we prove that for the systems with zero total charge it is possible to find a point $\boldsymbol{O}^{\prime}$, with respect to which all the components of quadrupole moment tensor vanish. If the contribution of the quadrupole moment in the charge distribution is essential, the electric field of a system can be substituted by the field of a point dipole located at the point $\boldsymbol{O}^{\prime}$. It will be shown below that this method as applied to the boundary problems allows obtaining physically clear approximate analytical results that coincide with high degree of accuracy with numerical ones. Let us consider the system with zero total charge. If the origin is displaced by the vector $\boldsymbol{\delta}$, then we have

$$
\int \rho(\boldsymbol{r})(\boldsymbol{r}-\boldsymbol{\delta}) \mathrm{d} V=\int \rho(\boldsymbol{r}) \boldsymbol{r} \mathrm{d} V=\boldsymbol{d},
$$

where $\rho(\boldsymbol{r})$ is the charge density, $\boldsymbol{d}$ is the dipole moment of the distribution. Due to (1) the dipole moment is independent of the position of the origin of the coordinates. As to higher multipoles, their values generally saying depend on the position of the point with respect to which they are calculated. This means that in principle, by proper choice of the origin, all the components of the quadrupole moment tensor can be set equal to zero. Further, we consider for the longitudinal oscillations the case of axial symmetry with respect to $Z$-axis and displace the origin $\boldsymbol{O}$ by $\delta$ along the $Z$-axis to the point $\boldsymbol{O}^{\prime}$ in order to satisfy the condition

$$
D_{Z Z}^{\prime}=\int \rho(\boldsymbol{r})\left[2(z-\delta)^{2}-x^{2}-y^{2}\right] \mathrm{d} V=D_{Z Z}-4 d_{Z} \delta=0 .
$$

Since $D_{X X}=D_{Y Y}=-\frac{1}{2} D_{Z Z}[20]$ and $d_{X}=d_{Y}=0$, all the components of the tensor vanish, if we choose the magnitude of $\boldsymbol{\delta}$ as follows: 
$\delta=\frac{D_{Z Z}}{4 d_{Z}}$.

In case of transversal oscillations the electron gas in the spheres oscillates in the direction $X$ perpendicular to the axis $Z$. Thus, the displacements in both cases of longitudinal and transversal oscillations are determined by the dipole and quadrupole moments of the system with respect to the origin of coordinates $\boldsymbol{O}$. Below the electric field of a neutral system in an external non-uniform field will be substituted just by the field of a point dipole located at the point $\boldsymbol{O}^{\prime}$. It is clear that the presented approach has an advantage in solving of boundary problems when higher than quadrupole moments of charge distribution can be neglected. The introduced approach can be characterized as "eliminated quadrupole moment approximation" (EQMA).

Let us consider two metallic spheres coated by dielectrics with dielectric constants correspondingly $\varepsilon_{1}$ and $\varepsilon_{2}$, and embedded in media with dielectric constant $\varepsilon_{0}$. The dielectric functions of the metals are correspondingly $\varepsilon_{3}$ and $\varepsilon_{4}$. The distance between the centers of the spheres is $a$, and the radii $R_{1}$ of the metallic cores are equal to each other. As to the dielectric coatings they have the same radii $R$ (see Fig. 1).

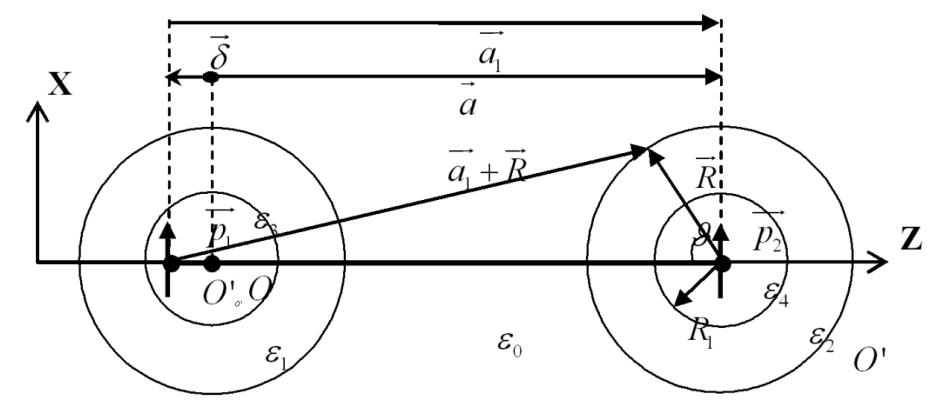

Fig. 1. Transversal dipoles, in-phase oscillations.

First consider transversal dipoles that oscillate in-phase and apply the EQMA method.

In the expansion of the electric field potential $\Phi$ of the first dipole placed at the point $\boldsymbol{O}^{\prime}$ in the vicinity of the center of the second one we keep only dipole and quadrupole terms

$$
\Phi=\frac{p_{1} r \sin \vartheta}{a_{1}^{3}}-\frac{3 p_{1} r^{2} \sin \vartheta \cos \vartheta}{a_{1}^{4}}+\frac{D_{2 X Z}}{r^{3}} \sin \vartheta \cos \vartheta,
$$

where $r$ is the distance from the center of the second sphere. Let us consider for simplicity of the calculations that the spheres are identical, i.e. the dielectrics and metals are the same. We search for the dipole and quadrupole moments of the second sphere with respect of its center in the induced potential (4) by solving the electrostatic boundary problem and obtain the following equations for $\varepsilon_{4}(x)$, 
where $x=\delta a^{-1}$ :

$$
\left\{\begin{array}{l}
\varepsilon_{4}(x)=\frac{\varepsilon_{2}}{2} \frac{6-2 \frac{3 \varepsilon_{2}+2 \varepsilon_{0}}{\varepsilon_{2}-\varepsilon_{0}} \frac{R_{1}^{5}}{R^{5}}+\left(3 \frac{3 \varepsilon_{0}+2 \varepsilon_{2}}{\varepsilon_{2}-\varepsilon_{0}}-6 \frac{R_{1}^{5}}{R^{5}}\right) \frac{a^{5}}{R^{5}} x(1-x)^{4}}{-2-\frac{3 \varepsilon_{2}+2 \varepsilon_{0}}{\varepsilon_{2}-\varepsilon_{0}} \frac{R_{1}^{5}}{R^{5}}+\left(-\frac{3 \varepsilon_{0}+2 \varepsilon_{2}}{\varepsilon_{2}-\varepsilon_{0}}-3 \frac{R_{1}^{5}}{R^{5}}\right) \frac{a^{5}}{R^{5}} x(1-x)^{4}}, \\
\varepsilon_{4}(x)=\varepsilon_{2} \frac{-2+\frac{\varepsilon_{0}+2 \varepsilon_{2}}{\varepsilon_{2}-\varepsilon_{0}} \frac{R_{1}^{3}}{R^{3}}+\left(-2 \frac{2 \varepsilon_{0}+\varepsilon_{2}}{\varepsilon_{2}-\varepsilon_{0}}+\frac{R_{1}^{3}}{R^{3}}\right) \frac{a^{3}}{R^{3}}(1-x)^{3}}{1+\frac{\varepsilon_{0}+2 \varepsilon_{2}}{\varepsilon_{2}-\varepsilon_{0}} \frac{R_{1}^{3}}{R^{3}}+\left(\frac{\varepsilon_{2}+2 \varepsilon_{0}}{\varepsilon_{2}-\varepsilon_{0}}+\frac{2 R_{1}^{3}}{R^{3}}\right) \frac{a^{3}}{R^{3}}(1-x)^{3}} .
\end{array}\right.
$$

Eliminating $x$ from Eqs. (5) it is possible to find the dependence of $\varepsilon_{4}$ on all the parameters $\varepsilon_{0}, R, R_{1}, \varepsilon_{2}$, and $a$. This procedure can be carried out numerically or graphically.

Counter-phase oscillations (when the dipoles have opposite directions) as well as both cases of the longitudinal oscillations can be described in the way presented above.

\section{Results}

After plotting two functions $\varepsilon_{4}(x)$ (Eq. (5)), we find the point of intersection. Finding $\varepsilon_{4}$ as ordinate of this point, it is easy to find the surface plasmon frequency from the following equation [20]:

$$
\omega(x)=\frac{\omega_{\mathrm{p}}}{\sqrt{\varepsilon_{d}-\varepsilon_{4}(x)}},
$$

where $\omega_{\mathrm{p}}$ is the bulk plasma frequency and $\varepsilon_{d}$ is the $d$-electron contribution into the dielectric function of the metal, determined experimentally [21].

We compare our calculations with the experimental results presented in [6]. In Fig. 2 we represent graphically the results of our calculations for in-phase transversal and longitudinal oscillations. Despite the fact that we do not dis-

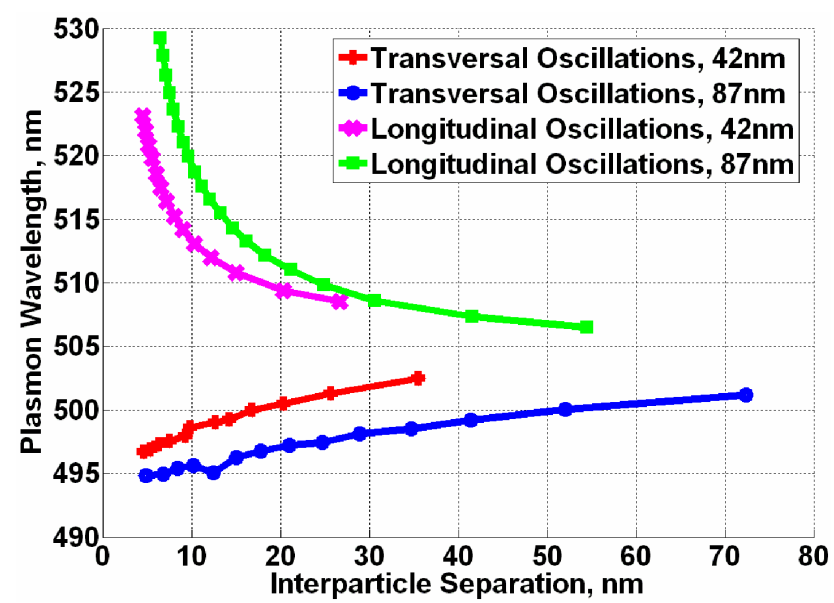

Fig. 2. The dependence of the surface plasmon frequencies on interparticle distance for in-phase transversal and longitudinal oscillations. 
cuss in this study the role of substrate under the particles, an obvious similarity in corresponding functions is present. The metal spheres ( $\mathrm{Au}$ in this case) with diameters $42 \mathrm{~nm}$ and $87 \mathrm{~nm}$ are covered by $4 \mathrm{~nm}$ shells with average dielectric constant equal to 2.56 , and the dielectric constant of the matrix is 2.25 . In case of longitudinal oscillations the $87 \mathrm{~nm}$ core lies above the $42 \mathrm{~nm}$ core in qualitatitave correspondence with the experimental results of $[6]$. We note that the general behaviour of the SP wavelength dependence on interparticle separation coincides with that of $[6]$.

\section{Acknowledgments}

This work was supported by the SCOPES grant \# IB 7320-111050.

\section{References}

[1] K.L. Kelly, E. Coronado, L.L. Zhao, G.C. Schatz, J. Phys. Chem. B 107, 668 (2003).

[2] E. Hao, G.C. Schatz, J. Chem. Phys. 120, 357 (2003).

[3] A. Dellafiore, F. Matera, F.A. Brieva, Phys. Rev. B 61, 2316 (2000).

[4] W. Ekardt, Phys. Rev. B 31, 6360 (1985).

[5] K.-H. Su, Q.-H. Wei, X. Zhang, J.J. Mock, D.R. Smith, S. Shultz. Nano Lett. 3, 1087 (2003).

[6] B.M. Reinhard, M. Siu, H. Agarwal, A.P. Alivisatos, J. Liphardt, Nano Lett. 5, 2246 (2005).

[7] S.A. Maier, M.L. Brongersma, P.G. Kik, H.A. Atwater, Phys. Rev. B 65, 193408 (2002).

[8] D.J. Bergman, Phys. Rev. B 19, 2359 (1979).

[9] M. Schmeits, L. Dambly, Phys. Rev. B 44, 12706 (1991).

[10] T. Atay, J.-H. Song, A.V. Nurmikko, Nano Lett. 4, 1627 (2004).

[11] A. Callegari, D. Tonti, M. Chergui, Nano Lett. 3, 1565 (2003).

[12] J. Aizpurua, G.W. Bryant, L.J. Richter, F.J. Garcia de Abajo, B. Kelley, T. Mallouk, Phys. Rev. B 71, 235420 (2005).

[13] J.J. Xiao, J.P. Huang, K.W. Yu, Phys. Rev. B 71, 045404 (2005).

[14] J.J. Penninkhof, A. Polman, L.A. Sweatlock, S.A. Maier, H.A. Atwater, A.M. Vredenberg, B.J. Koi, Appl. Phys. Lett. 83, 4137 (2003).

[15] S.Y. Park, D. Stroud, Phys. Rev. B 69, 125418 (2004).

[16] J.P. Huang, K.W. Yu, G.Q. Gu, Phys. Rev. E 65, 021401 (2002).

[17] R. Fuchs, Phys. Rev. B 11, 1735 (1975).

[18] R. Ruppin, Phys. Rev. B 26, 3440 (1982).

[19] W. Rechberger, A. Hohenau, A. Leitner, J.R. Krenn, B. Lamprecht, F.R. Aussenegg, Opt. Commun. 220, 137 (2003).

[20] M. Scharte, R. Porath, T. Ohms, M. Aeschlimman, J.R. Krenn, H. Ditlbacher, F.R. Aussenegg, A. Liebsch, Appl. Phys. B, Lasers Opt. B 73, 305 (2001).

[21] P.B. Johnson, R.W. Christy, Phys. Rev. B 6, 4370 (1972). 